\title{
A Dual-Use Enterprise Context for Vehicle Design and Technology Valuation
}

Adam B. Cooper, Michael Kokkolaras and Panos Y. Papalambros University of Michigan 
All rights reserved. No part of this publication may be reproduced, stored in a retrieval system, or transmitted, in any form or by any means, electronic, mechanical, photocopying, recording, or otherwise, without the prior written permission of SAE.

For permission and licensing requests contact:

SAE Permissions
400 Commonwealth Drive
Warrendale, PA 15096-0001-USA
Email: permissions @ sae.org
Fax: $\quad 724-772-4891$
Tel: $\quad 724-772-4028$

Tel: $\quad 724-772-4028$

For multiple print copies contact:

SAE Customer Service

Tel: $\quad$ 877-606-7323 (inside USA and Canada)

Tel: $\quad$ 724-776-4970 (outside USA)

Fax: $\quad$ 724-776-1615

Email: CustomerService@sae.org

\section{ISBN 0-7680-1319-4}

\section{Copyright $\odot 2004$ SAE International}

Positions and opinions advanced in this paper are those of the author(s) and not necessarily those of SAE. The author is solely responsible for the content of the paper. A process is available by which discussions will be printed with the paper if it is published in SAE Transactions.

Persons wishing to submit papers to be considered for presentation or publication by SAE should send the manuscript or a $\mathbf{3 0 0}$ word abstract of a proposed manuscript to: Secretary, Engineering Meetings Board, SAE.

\section{Printed in USA}




\title{
A Dual-Use Enterprise Context for Vehicle Design and Technology Valuation
}

\author{
Adam B. Cooper, Michael Kokkolaras and Panos Y. Papalambros \\ University of Michigan
}

Copyright $@ 2004$ SAE International

\begin{abstract}
Developing a new technology requires decisionmakers to understand the technology's implications on an organization's objectives, which depend on user needs targeted by the technology. If these needs are common between two organizations, collaboration could result in more efficient technology development. For hybrid truck design, both commercial manufacturers and the military have similar performance needs. As the new technology penetrates the truck market, the commercial enterprise must quantify how the hybrid's superior fuel efficiency will impact consumer purchasing and, thus, future enterprise profits. The Army is also interested in hybrid technology as it continues its transformation to a more fuel-efficient force. Despite having different objectives, maximizing profit and battlefield performance, respectively, the commercial enterprise and Army can take advantage of their mutual needs. Developing the new technology in a dual-use context allows the Army to leverage the design and production capabilities of the commercial enterprise, while the enterprise increases its hybrid production volume with military trucks during the low demand phase of hybrid market penetration.
\end{abstract}

This article describes the valuation of hybrid technology from both the enterprise and military perspectives using a previously developed enterprise decision model, which utilizes comprehensive vehicle simulation to drive decision-making. The enterprise is represented in a mathematical formulation that simultaneously optimizes initial vehicle design, product pricing, operating costs associated with capacity investment and design decisions, and the value created by the new products. The application of hybrid technology in the medium truck market illustrates the impact of dual-use decision-making on the commercial enterprise and satisfaction of military performance targets.

\section{INTRODUCTION}

The dual-use concept refers to joint development of technologies or products between government and commercial manufacturers to create value for both parties. This notion relates to the $21^{\text {st }}$ Century Truck Initiative, which is the transformation of the U.S. Army to a lighter, more mobile force while helping a commercial manufacturer develop and reduce costs of new technologies [1]. If both the military and commercial enterprise are interested in using the same technology in their respective fleets, they can take advantage of dual-use synergies.

Multiple examples of the dual-use initiative between the Army and industry are being implemented. The "SmarTruck," developed by the Army's National Automotive Center (NAC), is a light truck that has telematics, safety, and non-lethal weapon systems technology, which meet Army needs while utilizing the commercial Ford F-350 pickup truck chassis. Telematics, such as GPS and on-board communication, and safety technologies developed for the SmarTruck also benefit commercial users. The proliferation of these technologies benefits the commercial developer by creating a business case for selling cutting edge technology to new and returning customers [2].

The Army has extensively researched hybrid technology use in its new fleet of military vehicles. Logistical advantages of hybrid electric propulsion systems for fuel economy improvement, platform commonality, and performance were addressed for maintaining and supporting the Army's Future Combat System (FCS) force [3]. Another collaborative project is the Commercially Based Tactical Truck (COMBATT) being jointly developed by NAC and the DaimlerChrysler Hybrid Electric Vehicle Platform Engineering Group. In addition to utilizing a commercial light truck chassis, the COMBATT 
features a hybrid propulsion system incorporating an integral auxiliary power supply [4]. Another demonstration is a hybrid electric Army heavy truck built on a Class 8 motorhome chassis. In this application, the hybrid electric powertrain shares 90 percent of its components with a hybrid city bus [5].

The dual-use scenario described in this article assumes the Army awards a contract for medium hybrid truck production to a commercial enterprise and, in return, the Army receives closer to commercial truck prices.

A decision-making framework, which considers the design and market potential for a new technology over time and the commercial enterprise's production and marketing capabilities, is utilized to show the impact of joint development between the military and industry. This framework, called an Enterprise Decision Model, was previously developed for optimal vehicle design and technology valuation in the commercial market [6].

In the following, we explain the organizational structure of the enterprise, outline the models used in the decision-making framework for dual-use technology valuation, and demonstrate an application for the design and production of commercial and military medium hybrid trucks. We conclude with results and discussion of our findings.

\section{ENTERPRISE STRUCTURE}

Hybrid technology is assumed to be well developed by the enterprise for production in the commercial market. The enterprise, which is modeled after International Truck and Engine Inc., is assumed to have already won the bid for a military contract and is faced with two key decisions before continuing development: (i) choose the hybrid design most profitable based on the product's design and market capabilities and military performance specifications; and (ii) invest in capacity for hybrid production. We assume the enterprise chooses to invest in new hybrid capacity to avoid cannibalizing the current product [7].

In evaluating these decisions, we consider two organizational structures for product development. The first is a "disjoint" case in which design and production decisions are part of two independent divisions of the enterprise: commercial and military development, as illustrated in Figure 1.

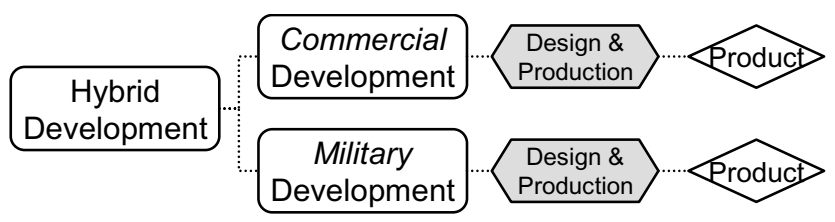

Figure 1. Disjoint design and production organizational structure for hybrid product development

The second alignment of the enterprise's resources is joint production of the military and commercial trucks (see Figure 2) because these products share many components. This alignment potentially takes full advantage of dual-use synergies.

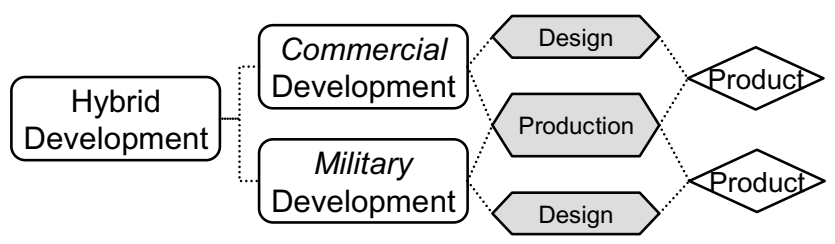

Figure 2. Commercial and military development sharing production resources (dual-use)

Decision-making and the subsequent value created using these two enterprise structures are compared to reveal the impact of dual-use development using the Enterprise Decision Model, discussed next.

\section{ENTERPRISE DECISION MODELS}

The Army believes hybrid technology is important for its transformation to a lighter, more mobile force because of the technology's capabilities of improving fuel efficiency and emissions, and supplying on-board power to the truck's operating systems. Commercial manufacturers are interested in hybrids for much of the same reasons. There is, of course, more uncertainty in the commercial market because manufacturers cannot be certain how consumers will react to the new, more expensive technology and how producing hybrid trucks will affect the enterprise's resource capabilities.

The following sub-sections summarize how interests of both the Army and enterprise are modeled. This decision-making framework is detailed more rigorously in Cooper and Papalambros [6].

\section{DEMAND, PRICING, AND PRODUCTION COSTS}

The enterprise must forecast future market penetration for hybrid technology for the beginning stages of hybrid production to make informed design, pricing, and production decisions today. The Utterback-Abernathy model for innovation, suggested to be an S-curve [8], is used to estimate the market 
penetration of hybrid technology over the technology's market life. This model is used in conjunction with Brownian motion, a technique that introduces future demand uncertainty by using the growth, volatility, and cyclical tendencies of the enterprise's historical sales data [8], to forecast the enterprise's hybrid demand over a 60-month production period. Brownian motion is used to randomly generate 100,000 demand forecasts for the commercial hybrid market, the average of which is shown in Figure 3.

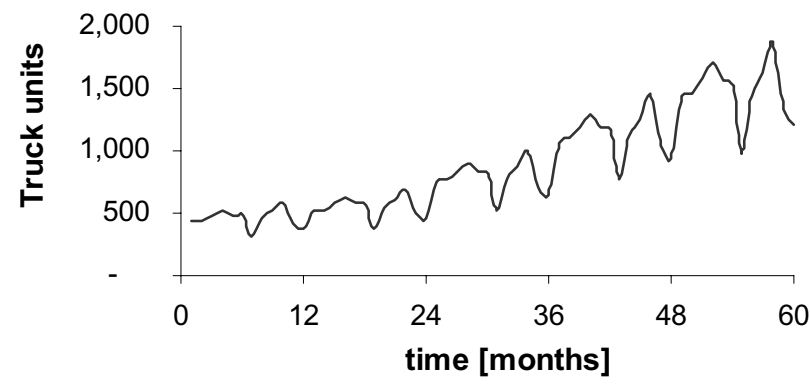

Figure 3. Forecasted commercial demand for medium hybrid trucks using Brownian motion and Utterback-Abernathy model

The commercial product's price $P$ relates to the enterprise's demand by the inverse demand curve,

$$
P=\alpha_{1}-\alpha_{2} Q^{D},
$$

where $Q^{D}$ is the Brownian motion demand forecast and the $\alpha_{1}$ and $\alpha_{2}$ parameters, regressed from historical data, describe consumer's price sensitivity.

As specified by the dual-use scenario, the Army expects truck prices closer to commercial prices. It is thus assumed the military truck price is a function of the base commercial price from Eq. (1) and a profit margin paid for hybrid component costs, detailed later. The military truck price $P_{M}$ becomes,

$$
P_{M}=\alpha_{1}-\alpha_{2} Q^{D}+\frac{L_{M}}{1-P M},
$$

where $L_{M}$ is the hybrid component learning curve cost and the profit margin $P M$ is assumed to be $10 \%$. From Eq. (2) the military's price is a function of the hybrid component cost, which is a function of the hybrid design.

Production costs are modeled using the current cost structure of International Truck and Engine Inc. because the cost of the hybrid truck is relatively the same as the conventional truck [10], except for hybrid component cost. The production cost is assumed to be a function of the utilization of the enterprise's hybrid capacity, given by

$$
C_{P}=48201-42253\left(\frac{Q^{P}}{K}\right)+25560\left(\frac{Q^{P}}{K}\right)^{2}
$$

with cost coefficients regressed from historical cost data and commercial hybrid units produced $Q_{C}^{P}$ defined by

$$
Q_{C}^{P}=\min \left(Q_{C}^{D}, K\right),
$$

which ensures the enterprise meets hybrid demand $Q_{C}^{D}$ if capacity $K$ is available. The minimum production cost from Eq. (3) is at $83 \%$ utilization, but the enterprise is assumed to invest in capacity today, which remains fixed during the 60-month production period because it is impractical to continuously adjust total capacity to meet changing demand [11]. Investing in capacity able to produce 2000 units per month allows the enterprise to capture all future demand (see Figure 3), but results in rather low utilization corresponding to higher production costs from Eq. (3). Less capacity means missing some sales, but lowering production costs. This tradeoff is very important for the capacity investment decision.

For the dual-use case, which assumes the enterprise shares production capabilities for the commercial and military trucks (see Figure 2), Eq. (3) becomes

$$
C_{P}=48201-42253\left(\frac{Q_{C}^{P}+Q_{M}^{P}}{K}\right)+25560\left(\frac{Q_{C}^{P}+Q_{M}^{P}}{K}\right)^{2},
$$

where the military quantity produced $Q_{M}^{P}$ is defined by the Army truck demand $Q_{M}^{D}$ as specified in the contract:

$$
Q_{M}^{P}=Q_{M}^{D}
$$

\section{PRODUCT DESIGN}

The central component of the EDM is to provide insight to the market and battlefield potential for the commercial and military trucks, respectively, based on the product's design capabilities. Design of the commercial and military hybrid trucks is represented using the hybrid electric version of Vehicle-EngineSIMulation (VESIM) [12], an advanced vehicle simulation model, configured as a parallel hybrid using a NiMH battery with an electric motor positioned after the transmission.

This high-fidelity vehicle simulation tool provides the relation between design characteristics and product performance using a combined Environmental Protection Agency (EPA) urban and highway driving cycle for the commercial truck. This combined cycle 
(see Figure 4), plus a more rigorous off-road cycle, is used for the military truck.

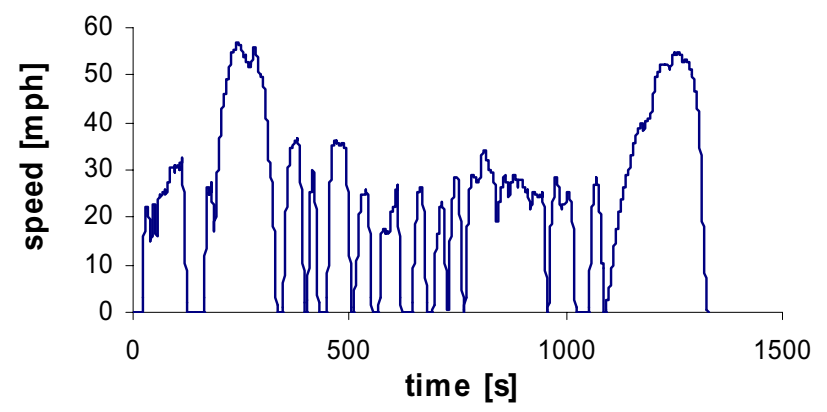

Figure 4. Urban and highway driving cycle for commercial truck and military support vehicle

Simulating the truck's design over these driving cycles determines the truck's fuel economy and acceleration performance.

Fuel economy is the most important product attribute for both commercial and military users. The enterprise can determine the present value of fuel cost savings $S_{C}$ for commercial consumers by discounting future fuel savings by the inflation rate $r_{i}$ using,

$$
S_{C}=\int_{0}^{240}\left[\frac{D_{t} M_{t}}{f e_{0}}-\frac{D_{t} M_{t}}{f e_{C}^{\prime}}\right] e^{-r_{i} t} d t
$$

Monthly diesel fuel price $D_{t}$ is forecasted using a mean-reverting process [9] with historical fuel price data and the truck's mileage $M_{t}$ is the EPA's 20-year life cycle of medium trucks [13]. The fuel economies, $f e_{0}$ and $f e_{c}^{\prime}$, are of the conventional baseline and proposed hybrid designs, respectively. Truck consumers are assumed to pay extra for increased fuel savings, so the price for the commercial truck from Eq. (1) becomes

$$
P=\alpha_{1}-\alpha_{2} Q^{D}+\frac{\Delta P}{\Delta S} S_{C},
$$

where $\Delta P / \Delta S$ is the portion of fuel savings that can be kept by the enterprise as a price premium, which is further described later.

\section{TECHNOLOGY COSTS}

The standard learning curve theory, namely, cost per unit decreases as a function of cumulative production $Q^{P_{z}}$ [14], is used to describe the decrease in cost of the new technology over the production period. The standard form of the learning curve is a power function:

$$
L=C_{H}\left(Q^{P}\right)^{-b},
$$

where $L$ is the learning curve cost, $C_{H}$ is the initial component cost of the hybrid design, and $b$ is a parameter that measures the rate of cost reduction as output increases. Hybridization cost $C_{H}$ is a function of the hybrid component design, as detailed in Cooper and Papalambros [6], and thus provides a tradeoff between design and cost.

For the dual-use case of joint hybrid production (see Figure 2), Eq. (9) becomes

$$
\begin{aligned}
& L_{C}=\left(C_{H}\right)_{C}\left(Q_{C}^{P_{\Sigma}}+Q_{M}^{P_{z}}\right)^{-b} \\
& L_{M}=\left(C_{H}\right)_{M}\left(Q_{C}^{P_{2}}+Q_{M}^{P_{2}}\right)^{-b} .
\end{aligned}
$$

Note that $C_{H}$ depends on the design of each individual truck, thus there are two cost equations in Eq. (10). Equations (9) and (10) provide insight to the advantages of sharing production capabilities for commercial and military trucks. The magnitude of hybrid truck production allows the enterprise to get down the learning curve faster, thus decreasing costs. For the military, decreased learning curve costs results in lower trucks prices, via Eq. (2).

\section{CAPACITY INVESTMENT AND VALUATION}

As previously discussed, the hybrid capacity investment decision has serious ramifications on the future cost and production capabilities of the enterprise. Another important tradeoff is that larger capacity, meant to capture more demand, results in greater cost to the enterprise today. The capacity investment cost $I_{K}$ is modeled by

$$
I_{K}=\rho(12 K)
$$

with a linear cost coefficient $\rho$ [15], approximated from previous enterprise capacity investments to be $\$ 12,425$ [16] per monthly unit of capacity $K$.

The final step in valuing the joint development of new technology is determining if the investment cost in Eq. (11) creates value to the enterprise. Net present value (NPV) is utilized to assess if future profits resulting from commercial and military production exceed the capacity investment cost required today. The objective of the Enterprise Decision Model is to maximize

$$
N P V=-I_{K}+\int_{T} \Pi_{C} e^{-r_{W A C C} t} d t+\int_{T} \Pi_{M} e^{-r_{f} t} d t
$$

where the profits $\Pi_{C}$ and $\Pi_{M}$ of the commercial and military products, respectively, are price minus cost 
times quantity produced, calculated from Eqs. (2) (3), (4), (8), and (9). The discount rate $r_{W A C C}$ in Eq. (12) is the weighted average cost of capital of the enterprise's assets, used to discount future profits in today's terms to compare to the investment cost [17]. The risk-free rate $r_{f}$ is used to discount military profits in Eq. (12) because the Army contract is guaranteed once agreed upon, and therefore carries low risk to the enterprise.

We assume the development period for the new products and building capacity is 12 months. Operational expenditures such as marketing and sales are not taken into account.

\section{DUAL-USE CASE STUDY}

The models described in the previous section are applied to a case study that investigates the effect of dual-use technology development on design and investment decisions, and the subsequent value created to the enterprise and Army. The enterprise, a truck manufacturer with established demand for conventional trucks, has developed hybrid technology for medium commercial $(C)$ and military $(M)$ hybrid trucks. The enterprise is assumed to invest in new capacity for hybrid truck production to avoid cannibalizing the conventional product in the commercial market.

The Army contract requires the enterprise to design and produce according to Army specifications. The details of this contract are assumed to be:

i. 50,000 medium hybrid trucks produced over a five-year period.

ii. Fuel economy target of $30 \%$ improvement over the conventional design and a performance constraint requiring acceleration time $0-45 \mathrm{mph}$ less than 16 seconds.

iii. Acquisition budget of $\$ 2$ billion.

The fleet size of 50,000 medium trucks is approximately one-fifth of the Army's tactical vehicle fleet being transformed to more fuel-efficient vehicles [1] and the budget is taken from a recent contract for medium military truck production over five years [18].

The optimization framework for the dual-use Enterprise Decision Model follows.

\section{DECISION VARIABLES}

Three design variables, engine displacement, hybrid motor scaling, and battery size, represent the technical design of the trucks. These variables are chosen to be off-the-shelf or discrete decisions because in practice, designers usually have only the option of certain size or specifications of design characteristics. The engine displacements in Table 1 are from a set of available engine sizes offered by Caterpillar [19]. The motor size, which is scaled up or down from a baseline motor, and battery size represent the hybrid system design and are used to calculate the cost of hybrid components $C_{H}$ used in Eq. (10).

Table 1. Off-the-shelf engineering design choices

\begin{tabular}{c|c}
\hline Design Variables & Available Choices \\
\hline displacement $[\mathrm{L}]$ & $\{9.6,10.3,12.0,14.6,15.8\}$ \\
motor scaling & $\{75 \%, 87.5 \%, 100 \%, 112.5 \%, 125 \%\}$ \\
battery size [modules] & $\{25,26,27, \ldots, 98,99,100\}$ \\
\hline
\end{tabular}

These design variables are input to VESIM to calculate the fuel economy and acceleration performance for each truck design.

Equation (8) assumes the enterprise passes a price premium for the consumer fuel savings $S_{C}$ resulting from the commercial hybrid design. The portion of fuel savings kept by the enterprise, $\Delta P / \Delta S$, is a marketing decision.

Recall the Army specifies a $30 \%$ improvement in fuel economy. This is modeled using

$$
\left|f e_{M}^{\prime}-f e_{0}(1.30)\right| \leq \varepsilon_{M},
$$

where $f e_{M}^{\prime}$ and $f e_{0}$ are the fuel economies of the military's hybrid and conventional baseline designs, respectively. Product designers attempt to minimize the deviation $\varepsilon_{M}$ from the Army's fe target.

The final decision is to invest in capacity $K$ for hybrid production. This decision impacts the investment cost (Eq. (11)) and commercial quantity produced (Eq. (4)). The military contract requires the enterprise to invest in at least 834 monthly units of hybrid capacity, which is the monthly production of 50,000 military trucks over 60 months.

\section{CONSTRAINTS}

For the commercial truck, we assume the design must meet at least current performance attributes. The acceleration performance constraints for $0-55$ and 30 $50 \mathrm{mph}$ (slightly relaxed to allow the optimizer to achieve feasibility more readily) are

$$
\begin{aligned}
t_{0-55} & \leq 20 \text { seconds } \\
t_{30-50} & \leq 10 \text { seconds. }
\end{aligned}
$$


The performance constraint for the military truck as specified by the contract is

$$
t_{0-45} \leq 16 \text { seconds. }
$$

The price premium decision $\Delta P / \Delta S$ of course depends on the consumer's willingness-to-pay for fuel savings. The hybrid pricing decision is constrained by a utility threshold [20], determined from enterprise marketing studies to be $\$ 5,000$. Therefore, the constraint is

$$
S_{C}-\Delta P_{C} \geq \$ 5,000
$$

That is, change in fuel savings (Eq. (7)) from the commercial hybrid design must exceed the change in price by at least $\$ 5,000$.

The enterprise makes design decisions for the military truck (Eqs. (13) and (15)) within the constraint of the military budget, modeled as

$$
\int_{12}^{72}\left(P Q^{P}\right)_{M} e^{-r_{t} t} d t \leq \$ 2.0 \text { billion . }
$$

"Army spending" created by military production, price $P_{M}$ times quantity produced $Q_{M}^{P}$, is discounted using the risk-free rate $r_{f}$ because the price of the military truck is time-dependent from Eq. (2) and (9).

\section{OPTIMIZATION FORMULATION}

The dual-use Enterprise Decision Model is summarized as follows.

$\max \left[-I_{K}+\int_{12}^{72}\left(\Pi_{C}\right) e^{-r_{W A C C} t} d t+\int_{12}^{72}\left(\Pi_{M}\right) e^{-r_{f} t} d t\right]-\varepsilon_{M}$

w.r.t. (engine displacement, motor \& battery size) ${ }_{C}$, (engine displacement, motor $\&$ battery size $)_{M}$,

$K, \Delta P / \Delta S, \varepsilon_{M}$

s. t. $S_{C}-\Delta P_{C} \geq \$ 5,000$

$\left(t_{0-55}\right)_{C} \leq 20$ seconds

$\left(t_{30-50}\right)_{C} \leq 10$ seconds

$\left(t_{0-45}\right)_{M} \leq 16$ seconds

$\left|f e_{M}^{\prime}-f e_{0}(1.30)\right| \leq \varepsilon_{M}$

$\int_{12}^{72}\left(P Q^{P}\right)_{M} e^{-r_{t} t} d t \leq \$ 2.0$ billion
DIRECT, a global optimization algorithm [21] is used to solve Eq. (18) because of its ability to treat mixeddiscrete variables. The NPV in Eq. (18) is scaled to the same magnitude as $\varepsilon_{M}$ for computational purposes.

The solution to the Enterprise Decision Model in Eq. (18) determines the optimal designs for the commercial and military trucks by considering joint development between the Army and commercial enterprise.

\section{RESULTS AND DISCUSSION}

The following sections discuss the results of the dualuse study. The first section compares the results generated from the two enterprise structures in Figures 1 and 2. The second section details the implications of the Army's assumed budget on achieving desired performance targets.

\section{ENTERPRISE STRUCTURE COMPARISON}

Comparison of commercial design and performance results between the disjoint and dual-use case is shown in Table 2.

Table 2. Commercial design and performance results

\begin{tabular}{c|cc}
\hline \multirow{2}{*}{$\begin{array}{c}\text { Decisions and } \\
\text { Responses }\end{array}$} & \multicolumn{2}{|c}{ Commercial } \\
& Disjoint & Dual-use \\
\hline displacement & 9.6 & 9.6 \\
motor scaling & $-12.5 \%$ & $-25 \%$ \\
battery size & 36 & 25 \\
hybrid cost & $\$ 9,448$ & $\$ 7,590$ \\
$\% \Delta$ fuel economy & $26 \%$ & $23 \%$ \\
fuel cost savings & $\$ 15,929$ & $\$ 14,435$ \\
$t_{0-55}$ [sec] & 19.8 & 19.8 \\
$t_{30-50}$ [sec] & 8.9 & 8.8 \\
\hline
\end{tabular}

The military production in the dual-use case allows the enterprise to get down hybrid learning curve faster, via Eq. (10), versus the dual-use case. This reduction in cost leads decision-makers to design a less expensive hybrid system, as shown in Table 2, to maximize the value of the commercial product. The less expensive hybrid system relates to lower commercial prices to consumers due to the decrease in fe improvement. The following discussion helps put the reasoning for this design decision change into perspective.

Marketing and production decisions are shown in Table 3 for the commercial product along with the corresponding enterprise financial implications. 
Table 3. Enterprise decisions and value created from commercial market

\begin{tabular}{c|cc}
\hline $\begin{array}{c}\text { Decisions and } \\
\text { Implications }\end{array}$ & \multicolumn{2}{|c}{ Commercial } \\
Disjoint & $\begin{array}{c}\text { Dual- } \\
\text { use }\end{array}$ \\
\hline price premium (\%) & 68.5 & 64.8 \\
capacity [units/mo] & 654 & 841 \\
capacity investment [\$M] & 98 & 125 \\
present value [\$M] & 201 & 260 \\
\hline
\end{tabular}

Due to the decrease in fuel economy for the dual-use design, the enterprise must lower the price premium by passing $3.7 \%$ less of the fuel savings. This is countered, however, by the dual-use synergies encouraging decision-makers to invest an additional $\$ 27$ million or $28 \%$ in commercial capacity. Consequently, the enterprise captures $17 \%$ more of the commercial demand (see Figure 5) leading to an almost $30 \%$ increase in present value created by the commercial product.

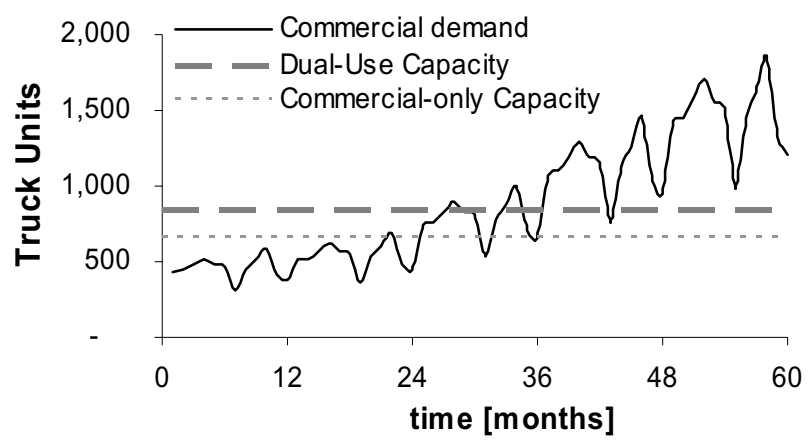

Figure 5. Commercial demand and optimized capacity levels for disjoint (commercial-only) and dual-use cases

This is an example of the tradeoff decision-makers face between pricing and production volume and more importantly, how product design decisions impact these non-technical decisions.

Comparison of military results between the disjoint and dual-use cases is shown in Table 4.

Table 4. Military design and performance results and Army spending

\begin{tabular}{c|cc}
\hline \multirow{2}{*}{$\begin{array}{c}\text { Decisions and } \\
\text { Responses }\end{array}$} & \multicolumn{2}{|c}{ Military } \\
& Disjoint & Dual-use \\
\hline displacement & 9.6 & 9.6 \\
motor scaling & $+25 \%$ & $+25 \%$ \\
battery size & 46 & 49 \\
hybrid cost & $\$ 11,882$ & $\$ 12,291$ \\
$\% \Delta$ fuel economy & $28.4 \%$ & $29.3 \%$ \\
acceleration 0-45 [sec] & 13.6 & 13.6 \\
Army spending [\$M] & $\$ 1,999.7$ & $\$ 1,987.2$ \\
\hline
\end{tabular}

From the military perspective, dual-use allows designers to achieve a fuel economy closer to the $30 \%$ target for the military truck $(29.3 \%$ vs. $28.4 \%)$ within the same $\$ 2$ billion budget. This is because the military's hybrid learning curve costs decrease faster for the dual-use case due to the production of commercial hybrids, thus reducing the military's truck price in Eq. (2), via Eq. (10), therefore allowing a more expensive hybrid configuration with increased fe. Interestingly, for the same reasons just described, the Army spends $\$ 12.5$ million less than the disjoint case despite the more expensive hybrid design and better fuel economy.

The final comparison is to reveal the monetary impact of dual-use synergies on the commercial enterprise. Table 5 shows the financial implications of each product for the disjoint cases and then combines them, which is representative of the value created by the organizational structure in Figure 1, to compare to the dual-use case.

Table 5. Comparing value created by disjoint cases versus dual-use case

\begin{tabular}{c|cc|c|c}
\hline \multirow{2}{*}{ Value } & \multicolumn{2}{|c|}{ Disjoint } & Disjoint & Dual-use \\
& Comm. & Military & Combined & \\
\hline$P V[\$ M]$ & 201 & 289 & 490 & 562 \\
$I_{K}[\$ M]$ & 98 & 125 & 223 & 250 \\
$N P V[\$ M]$ & 103 & 165 & 268 & 312 \\
\hline
\end{tabular}

Taking full advantage of dual-use synergies increases total value created (NPV) by $\$ 44$ million or $16 \%$ from just a $12 \%$ increase in hybrid capacity investment $\left(I_{K}\right)$.

\section{ARMY BUDGET IMPLICATIONS}

It is evident that the Army's budget is limiting the military design from achieving its goal. Following this, the military has two choices if meeting the $30 \%$ fe target is crucial to battlefield success: (i) decrease the number of trucks required (from 50,000), which may result in decreased battlefield capabilities, or (ii) increase its acquisition budget for medium trucks, which may limit resources for other military products. The latter is examined by increasing the Army's budget $12.5 \%$ to $\$ 2.25$ billion to show the budget's implications. The results are shown in Table 6 for the disjoint case only. 
Table 6. Comparing decisions and responses for increased Army budget

\begin{tabular}{c|c|c}
\hline $\begin{array}{c}\text { Decisions and } \\
\text { Responses }\end{array}$ & $\begin{array}{c}\$ 2.0 \text { Billion } \\
\text { Budget }\end{array}$ & $\begin{array}{c}\$ 2.25 \\
\text { Billion } \\
\text { Budget }\end{array}$ \\
\hline displacement & 9.6 & 9.6 \\
motor scaling & $+25 \%$ & $+12.5 \%$ \\
battery size & 46 & 87 \\
hybrid cost & $\$ 11,882$ & $\$ 17,122$ \\
$\% \Delta$ fuel economy & $28.4 \%$ & $30.0 \%$ \\
acceleration 0-45 [sec] & 13.6 & 13.7 \\
Army spending $[\$ \mathrm{M}]$ & $\$ 1,999.7$ & $\$ 2,242.5$ \\
\hline
\end{tabular}

By re-allocating its acquisition budget, the Army can successfully reach its performance target of $30 \%$ fuel economy improvement.

\section{CONCLUSIONS}

This analysis reveals the added benefit of the Army and commercial enterprise making decisions simultaneously. Dual-use technology valuation obviously benefits the military by permitting better product attributes within the same budget. From the commercial perspective, the enterprise can afford to capture more hybrid demand and increase total enterprise value as a result. The Enterprise Decision Model helps decision-makers make informed design, production, and marketing decisions to recognize and take full advantage of dual-use synergies.

\section{ACKNOWLEDGEMENTS}

We gratefully acknowledge P. Georgiopoulos for ideas contributed during the execution of this work. The research was partially supported by the US Army TACOM through the Automotive Research Center and a Dual Use Science and Technology Project at the University of Michigan. This support is gratefully acknowledged. The views presented here are only those of the authors and do not necessarily reflect views of the sponsors or other individuals we consulted.

\section{REFERENCES}

[1] Skalny, P.F., A.J. Smith, D. Powell, 2001. " $21^{\text {st }}$ Century Truck Initiative Support to the Army Transformation Process," SAE Int. SAE Paper 2001-01-2772.

[2] Kozera, M.J., G. Fuller-Simms, and D.J. Gorsich, 2001. "Telematics for 'SmarTruck'," SAE Int. SAE Paper 2001-01-2795.

[3] Ernat, J.S., W.G. Harris, and T. Trzaska, 2001. "Advanced Hybrid Electric Drive (AHED) Technology Demonstrator," SAE Int. SAE Paper 2001-01-2774.

[4] Almond, H., W. Doolittle, and G. Klees, 2001. "Hybrid Propulsion Truck Platform Dually Developed by the
U.S. Army and DaimlerChrysler," SAE Int. SAE Paper 2001-01-2794.

[5] Kluger, M.A., D. Szkubiel, and E.A. Bass, 2001. "A Dual-Use Hybrid Electric Command and Control Vehicle," SAE Int. SAE Paper 2001-01-2775.

[6] Cooper, A.B. and P.Y. Papalambros, 2003. "An Enterprise Decision Model for Optimal Vehicle Design and Technology Valuation," ASME 2003 IMECE, Washington, D.C. (in review).

[7] Cooper, A.B, 2003. An Enterprise Decision Model for Optimal Vehicle Design and Technology Valuation, M.Sc. Thesis, University of Michigan, Ann Arbor, Michigan.

[8] Afuah, A, 1998. Innovation Management, Oxford University Press, NY.

[9] Dixit, A.K., and R.S. Pindyck, 1994. Investment Under Uncertainty. Princeton University Press, Princeton, NJ.

[10] An, F., F. Stodolsky, A. Vyas, R. Cuenca, and J.J. Eberhardt, 2000. Scenario Analysis of Hybrid Class 3-7 Heavy Vehicles. SAE Int. SAE Paper 2000-01-0989.

[11] Morrison, C.J., 1993. A Microeconomic Approach to the Measurement of Economic Performance: Productivity Growth, Capacity Utilization, and Related Performance Indicators, Springer-Verlag, New York.

[12] Lin, C.-C., Filipi, Z.S., Wang, Y., Louca, L., Peng, H., Assanis, D.N., and J.L. Stein, 2001. "Integrated, FeedForward Hybrid Electric Vehicle Simulation in SIMULINK and its Use for Power Management Studies," SAE 2001 World Congress, Detroit, Michigan, SAE Paper 2001-01-1334.

[13] EPA, 2002. Nonconformance Penalties for 2004 Highway Heavy Duty Diesel Engines, Draft Technical Support Document. Environmental Protection Agency.

[14] Argote, L. and D. Epple, 1990. "Learning Curves in Manufacturing," Science, 247, 920-924.

[15] Dixit, A.K, and R.S. Pindyck, 2000. "Expandability, Reversibility, and Optimal Capacity Choice." Trigeorgis, L. and M.J. Brennan (Eds), 2000. Project Flexibility, Agency, and Competition, Oxford University Press, New York.

[16] Navistar, 1997. 10-k report, http://www.shareholder.com/nav

[17] Brealey, R.A., and S.C. Myers, 2000. Principles of Corporate Finance, Irwin/McGraw-Hill, Boston, MA.

[18] Stewart and Stevenson Inc., 2002. http://www.ssss.com/fmtv/

[19] Caterpillar, 2003. http:// www.caterpillar.com/

[20] Adner, R., and D. Levinthal, 2001. "Demand Heterogeneity and Technology Evolution: Implications for Product and Process Innovation," Management Sci. 47, 611-628.

[21] Jones, D., 1999. "DIRECT," Encyclopedia of Optimization, Kluwar Academic Publishers. 